\title{
NOUVELle
}

\section{L-dopa, \\ un jour et pour toujours}

Erwan Bezard
UMR CNRS 5227, Mouvement-Adaptation-Cognition, 146, rue Léo Saignat, 33076 Bordeaux Cedex, France. erwan.bezard@u-bordeaux2.fr
> La L-dopa, précurseur de la dopamine, constitue actuellement le médicament le plus utilisé dans le traitement de la maladie de Parkinson [1]. Mais ce traitement reste néanmoins très insatisfaisant en raison de ses effets secondaires importants. Parmi eux, et non des moindres, les dyskinésies, qui se traduisent notamment par l'émergence progressive de mouvements involontaires et incontrôlés ainsi que des fluctuations de la réponse au traitement [2].

Les patients ainsi traités voient donc bien diminuer leurs symptômes parkinsoniens mais connaissent de plus en plus de difficultés à effectuer des tâches quotidiennes. En collaboration avec des chercheurs suédois (P. Andren)

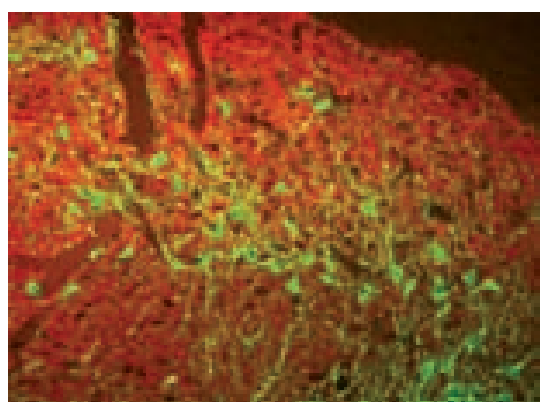

Neurones dopaminergiques de la substance noire (chez le rat) (๔ Photo Dr Steve Asmus, Centre College, Danville, Ky, États-Unis). et anglais (A.R. Crossman), nous venons de démontrer, chez le primate, que la toute première administration de L-dopa induit des changements définitifs dans le cerveau dès 1 heure après son ingestion. Les profils protéiques d'animaux traités une seule fois équivalent les profils d'animaux traités au long cours [3]. Si de tels mécanismes étaient subodorés, ils n'avaient jamais été démontrés jusqu'à présent [4, 5]. L'apparente équivalence entre un traitement aigu et un traitement chronique suggère que le phénomène « d'empreinte » serait la conséquence de la maladie elle-même (c'est-à-dire la perte de la dopamine), les administrations de L-dopa ne faisant qu'exacerber la sensibilisation du système et ne l'induisant pas. En outre, ces résultats signifient que les patients exposés à la L-dopa au cours du processus de diagnostic de leur maladie de Parkinson voient la réponse de leur cerveau fondamentalement modifiée dès ce tout premier traitement et ce de façon irrémédiable.

Ces travaux associant une modélisation extrêmement fine des stades cliniques chez le primate à une analyse protéomique à large échelle (gels bidi- mensionnels associés à la spectroscopie de masse) obligent à reconsidérer le développement de stratégies thérapeutiques antiparkinsonienne fondées sur le remplacement de la dopamine, voie encore actuellement poursuivie par l'industrie. Ils permettent cependant d'identifier un certain nombre de cibles thérapeutiques potentielles que nous allons maintenant nous attacher à valider en vue du développement de co-traitements permettant de continuer à utiliser la L-dopa. $\diamond$

Striatal proteomic analysis suggests that first L-dopa dose equates to chronic exposure

\section{RÉFÉRENCES}

1. Fahn S, Oakes D, Shoulson I, et al. Levodopa and the progression of Parkinson's disease. N Engl J Med 2004 ; $351: 2498-508$

2. Rascol 0 , Brooks DJ, Korczyn AD, et al. A five-year study of the incidence of dyskinesia in patients with early Parkinson's disease who were treated with ropinirole or levodopa. $N$ Engl J Med 2000 ; 342 : 1484-91.

3. Scholz B, Svensson M, Alm H, et al. Striatal proteomic analysis suggests that first $L$-dopa dose equates to chronic exposure. PLoS One 2008 ; 3 : el589.

4. Bezard E, Brotchie JM, Gross CE. Pathophysiology of levodopa-induced dyskinesia : potential for new therapies. Nat Rev Neurosci 2001 ; 2 : 577-88.

5. Cenci MA. Dopamine dysregulation of movement control in L-dopa-induced dyskinesia. Trends Neurosci $2007 ; 30: 236-43$.

ILLUSTRATION DES ARTICLES (VIGNETTES) : p. 339 : cellules nerveuses (photo Pascal Dournaud - @ Photothèque Inserm) - p. 340 : caryotype humain (photo Michel Depardieu - ( ) Photothèque Inserm) - p. 343 : œuf humain âgé de deux jours après fécondation (photo Jacques Testart - (c) Photothèque Inserm) - p. 344 : cellules de l'intestin grêle (photo Claudia Haffen - (c) Photothèque Inserm) - p. 344 : médicaments (photo Michel Depardieu - (c) Photothèque Inserm) - p. 348 et 352 : cristaux de rénine humaine (photo Jean-Paul Mornon - ( Photothèque Inserm) p. 349 : lésion d'athérome au niveau de la racine de l'aorte (@ photo Giuseppina Caligiuri) p.353: vue transversale d’ADN (photo Jean-Louis Martin - (C) Photothèque Inserm) - p. 375 : Phaeodactylum tricornutum exprimant la green fluorescent protein (๑ photo Aude Carlier) - p. 383 : première division méiotique de l'ovocyte de souris (๔ photo Katja Wassmann) - p. 390 : carie atteignant le sillon de la couronne dentaire (photo Bertrand Kerebel - ( ) Photothèque Inserm) - p. 399: culture primaire de neurones d’hippocampe de souris (๔ photo Norbert Weiss) p. 407 : adipocytes en culture (photo Michel Depardieu - (c) Photothèque Inserm). 